\title{
Curvature Measurement of Thin Plates with the Help of Digital Image Processing
}

\author{
Péter MÁTÉ, ${ }^{1}$ András SZEKRÉNYES ${ }^{2}$ \\ Budapest University of Technology and Economics, Faculty of Mechanical Engineering, \\ Department of Applied Mechanics, Budapest, Hungary \\ ${ }^{1}$ peter.mate@mm.bme.hu \\ ${ }^{2}$ szeki@mm.bme.hu
}

\begin{abstract}
The verification of different plate bending problems require a punctual measurement method of the bent shape. However, with a proper curvature measurement procedure the calculations can be made more accurate. This is due to the workaround this method provides by neglecting the inaccuracies of the beam theory and the tensile tests, measuring directly the function between the curvature and bending moment for a given sheet metal. The measurements in this paper are made with the help of a digital camera and telephotographic lens. The evaluations of these images are compared to the results obtained from the Euler-Bernoulli beam theory. While the results regarding the curvature measurements have a significant deviation, the shape of the plate is in good agreement with the numerical calculations.
\end{abstract}

Keywords: curvature, thin plate, bending, image processing.

\section{Introduction}

The widespread method for the calculation of the bent beam's or plate's shape is based on the curvature generated by the bending moment acting upon the cross section. The literature offers several models for the description of the relationship between the curvature and bending moment, however, these are based upon assumptions, and heavily rely on the uniaxial tensile test representing the material's behaviour. There were two goals with this experiment: on one hand was the development of a procedure through which the curvature distribution of a bent beam or plate can be measured, on the other hand it must offer a direct measurement of the curvature-bending moment function for a particular beam or plate. With this method one could skip the usage of a beam or sheet model and eliminate the effect of neglections and assumptions it uses

\section{Imaging}

The main objective of the imaging was to precisely map the projection curve of the mid-plane of the plate (i.e. centerline) onto a two-dimension- al raster. Taking this into account, thin sheet metal is extremely good for this measurement, since the thinner the plate, the more precisely the position of the centreline can be determined.

Ideally, one should use parallel projection for imaging, for which scanning is the closest widely available method. Scanning was used also by Kovalevsky (2001) to analyse the contour's curvature for various objects [1]. At the time of the measurements, no proper imaging device was on hand, thus a DSLR camera was used instead. This, considering the nature of the lens system, is not a perfect substitution, because the lenses can distort the picture as one diverges from the optical axis of the system. There are several more factors that reduce the precision of the image: for example, the fact that the plate has a depth parallel to the optical axis which together with the central projection makes the plate seem thicker in the picture. A further factor that negatively affects the precision is the lack of proper lighting: the plate has a reflection and a shadow on the background, which can blur the plate-background boundaries. 
Another factor is the resolution of the image sensor: the higher the resolution of the useful image, the higher the precision of the measurement. To eliminate these negative effects and still give the plate enough space on the picture a camera with telephotographic lens was used, with the narrowest possible aperture, and the highest possible zoom, so that the shape of the bent plate would fill almost the whole picture. The plate was painted blue and red paper used as background to make them easily distinguishable from each other. The image made and used throughout this article can be seen in Figure 1. The pictures were made with a CANON EOS 1000D camera and a 350mm Sigma lens.

\section{Image processing}

To process the image the HSB colour system was used, this was what determined the choice of colours for the plate and the background, since these two colours are well distinguishable on this colour scale.

The most important task is to properly separate and isolate the plate from the rest of the picture. With the lack of laboratory circumstances and a well-controlled imaging process, the first step is to manually delete everything from the picture that is not the plate and its narrow environment. Similarly, the second step is to examine each image separately (if there are more), after which some threshold values can be manually determined for the $H$ (hue), $S$ (saturation), $B$ (brightness). With the help of threshold values the pixels containing the plate can be filtered algorithmically. One such H-S-B distribution can be seen in Figure 2.

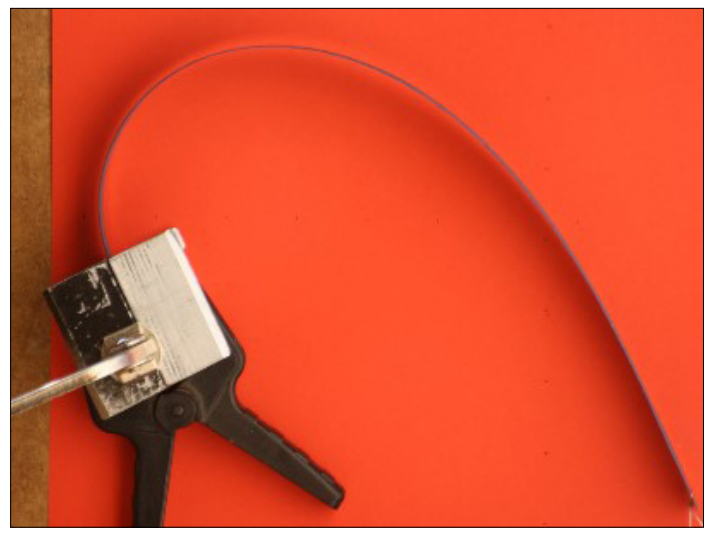

Figure 1. The picture used for the measurements (3888x2592 px).

\subsection{Image evaluation}

In Figure 2 blurring of the plate-background boundary can be seen: the position of the plate's edge is not clear. To solve this problem, one must produce a weight map from the pixels' colour data, the same way Kovalevsky [1] and Coeurjolly et. al. [2] did on grayscale images. While the grayscale value provides a natural weighting method, with colored images one needs to create a unique weighting procedure, based upon the investigation from Figure 2. In this way, one attributes a weight value to each pixel.

These values can be arranged into a matrix (w), which represents the weight map of the image. The calculation of this matrix is done according to equation (1), if the $H, S, B$ values are within the manually determined boundaries. In every other case the weight value is null.

$$
w_{i j}=\frac{H_{i j}}{S_{i j} \cdot B_{i j}}
$$

\subsection{Finding the centreline}

When searching for the coordinates of the centreline, The coordinates of the pixels which contain the plate were averaged with the help of the weight map. This can be done in two ways: averaging along the coordinate axes or with the help of polar coordinates, averaging along the radial coordinates. For the evaluation of the image the latter was used, since it gives a far smoother set of points than the calculations along the Descartes-coordinates. A qualitative representation of the cause of this phenomenon can be found in Figures 3. and 4.

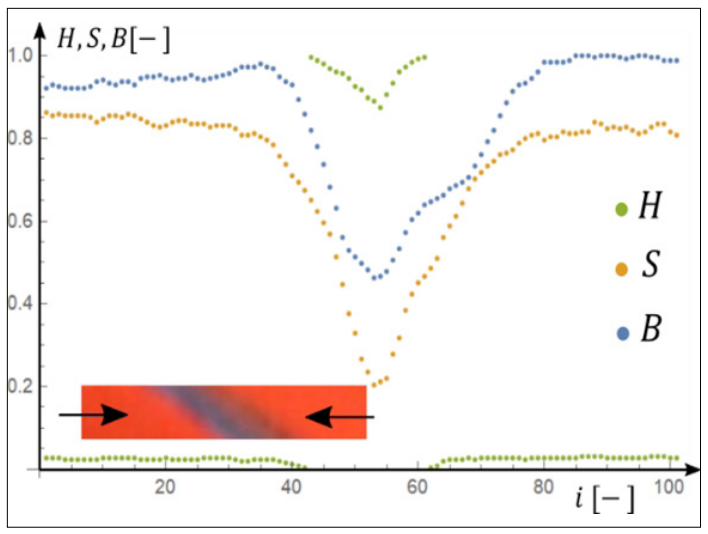

Figure 2. The distribution of the $H, S, B$ values of a pixel row as a function of the horizontal pixel-coordinate. 

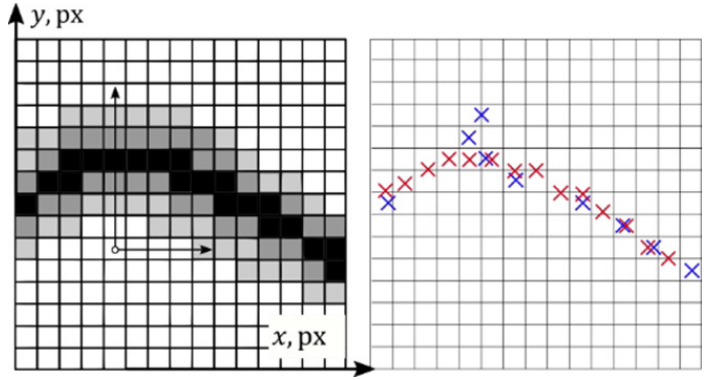

Figure 3. The averaging of the plate's coordinates along the $x$ and $y$ coordinate axes.

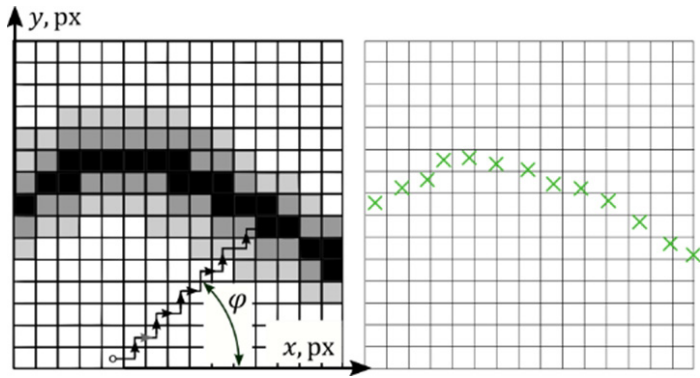

Figure 4. The averaging of the plate's coordinates in a quasi-polar system.

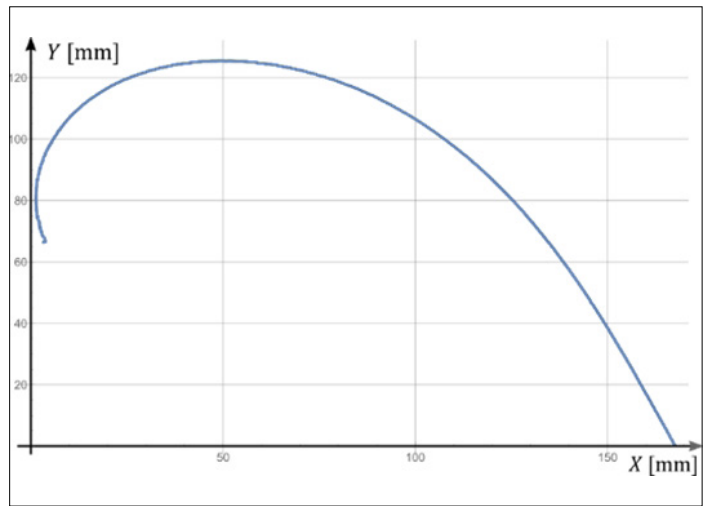

Figure 5. The raw point-set representing the centerline of the bent plate.

The computational procedure of the centreline coordinates in a polar system is presented in equation (2). The indexes of the $w$ matrix represent the $\left\{x_{i} ; y_{i}\right\}$ coordinates of the image. In the equation these appear as real numbers, thus when writing the algorithm, one must truncate them and take only their integer part into account.

$$
r_{i}=\frac{\sum_{j} r_{j} \cdot w_{r_{j} \cdot \cos \left(\varphi_{i}\right), r_{j} \cdot \sin \left(\varphi_{i}\right)}}{\sum_{j} w_{r_{j}} \cdot \cos \left(\varphi_{i}\right), r_{j} \cdot \sin \left(\varphi_{i}\right)}
$$

With these calculations one obtains $\left\{\varphi_{i} ; r_{i}\right\}$ coordinate-pairs, which have to be transformed back to the Descartes coordinate-system, to get the $\left\{x_{i} ; y_{i}\right\}$-coordinate-pairs of the centerline. In some cases, it is advised that the center of the polar coordinate-system be repositioned, to obtain better results while determining the position of the centerline. Ideally, one should always average in a direction that is perpendicular to the plate's mid-plane, but this is not discussed in this article

The conversion of the px unit into $\mathrm{mm}$ is achieved with control-lengths drawn onto the background of the plate. The converted coordinates measured in millimeters are marked by $\left\{X_{i} ; Y_{i}\right\}$ The raw set of points in the $X-Y$ coordinate-system can be seen in Figure 5. This consists of 5017 points.

\section{Calculating the curvature}

To calculate the curvature, one must use a numerical method since the bent shape is given only with separate points. For an analytically given $f: \mathbb{R} \rightarrow \mathbb{R}, f(x)$ function the calculation of the curvature $(\kappa)$ of the osculating circle can be done according to equation (3).

$$
\kappa=\frac{f^{\prime \prime}(x)}{\left(1+f^{\prime}(x)^{2}\right)^{3 / 2}}
$$

For a function numerically given, this equation can be used with the help of various numerical differentiation schemes, but these schemes are sensitive to the equidistant division of the point set [3]. This criterion is not met in our case, thus two invariant curvature calculating schemes were used, proposed by Dalle [3] and Belyaev [4]. In theory the invariant schemes show a first order convergence [3], though the points should be precise for it to work properly, which is not possible in this kind of measurement. Firstly, to eliminate the error of the raw centerline, data moving averages were used to smooth the curve, then the sampling window widened for curvature approximation.

\subsection{Curvature from three points}

One of the easiest invariant methods is to take the circumscribed circle's curvature of three consecutive points as an approximation. The illustration of the procedure can be seen in Figure 6. The curvature of the circle can be determined using equation (4), where $T_{\Delta}$ is the area of the triangle, which can be calculated using Heron's formula.

$$
\kappa \approx \frac{4 \cdot T_{\Delta}}{h_{1} \cdot h_{2} \cdot h_{3}}
$$




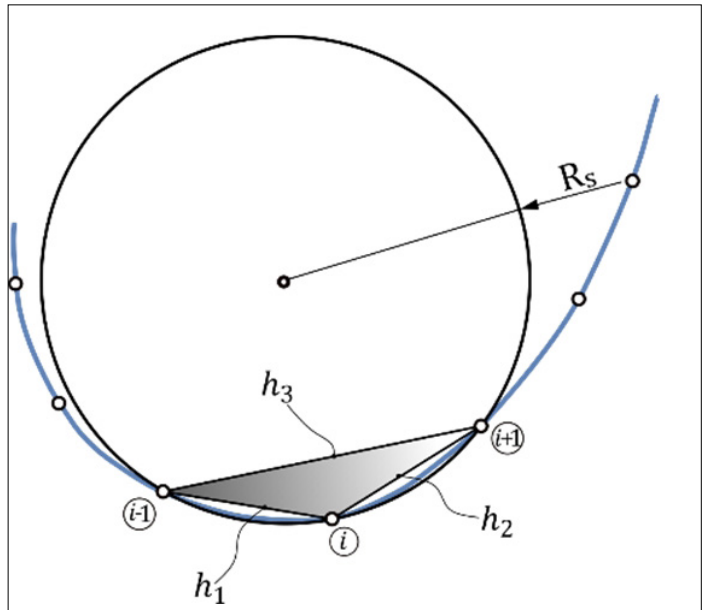

Figure 6. Approximating the curvature with the circumscribed circle

\subsection{Curvature from fitted function}

Because of the raw curve's noise, a considerable method is the local polynomial fitting with the method of least squares. The curvature is then approximated by the curvature of the polynomial in the middle of the selected window. For the best fitting of the function, one should rotate the point set so that its tangent is horizontal in the middle of the set. By increasing the polynomial's degree, the smoothness of the curvature function is reduced, thus the second-degree polynomial is the best for this application. According to the current work the circumscribed circle method and this method give the same results for the same sampling window.

\section{Discussion of the results}

Several attempts were made on the curvature calculations and raw data smoothing, the ideal combination can be seen in Figure 7. The smoothing and curvature calculating parameters are given in the figure. These are the window width for the moving average smoothing $\left(n_{s}\right)$, the number of smoothing procedures $\left(n_{\text {sim }}\right)$, and the sampling radius for the curvature calculations $(n)$. The least squares fit for the curvature results can also be seen in Figure 7.

The results were compared with those obtained from a numerical procedure developed earlier [5] Both the curvature function (7. ábra) and the shape (8. ábra) are in good agreement. The model used for the calculations can be seen in Figure 9, the input data is given in Table 1.

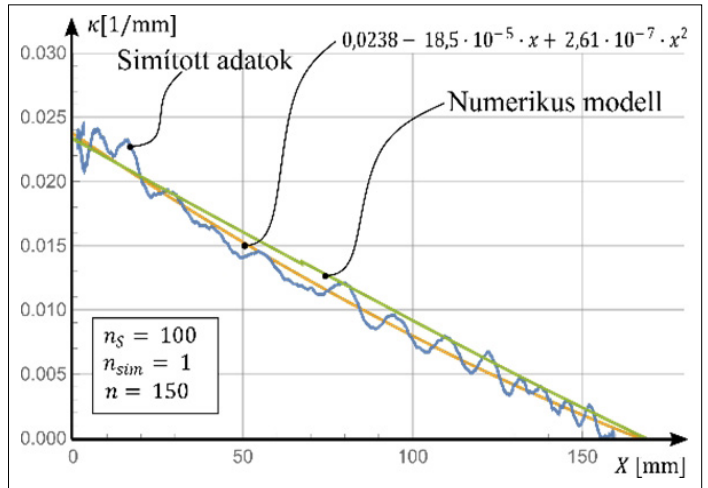

Figure 7. The measured (blue), the fitted (yellow) and calculated (green) curvature function of the plate.

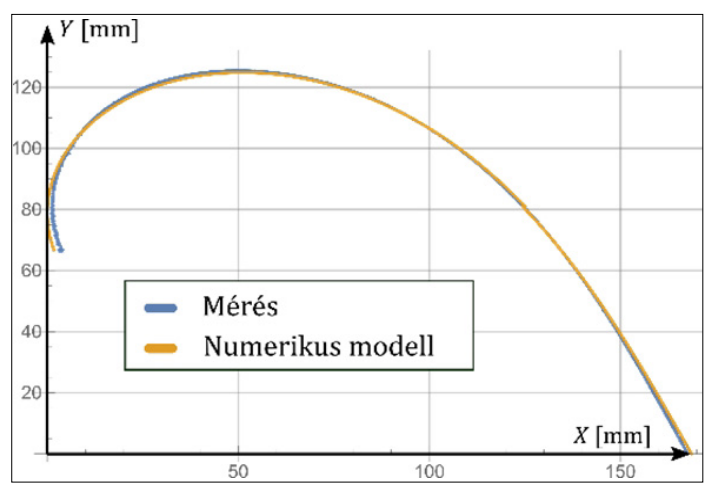

Figure 8. The measured and calculated shape of the plate.

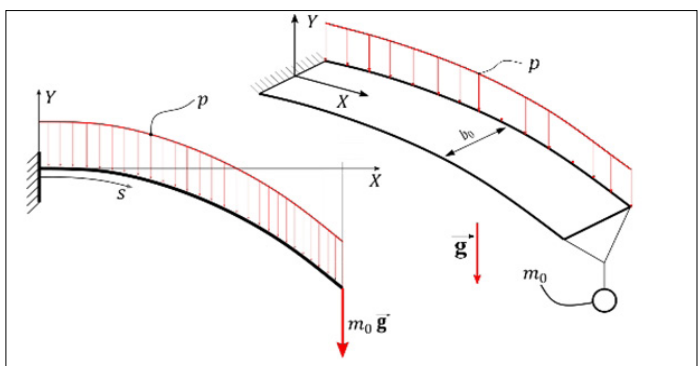

Figure 9. The mechanical model of the plate.

Table 1. Parameters for the numerical calculations

\begin{tabular}{|l|c|c|}
\hline \multicolumn{1}{|c|}{ Parameter } & Value & Unit \\
\hline Modulus of elasticity & 200 & $\mathrm{GPa}$ \\
\hline Poisson's ratio & 0.33 & - \\
\hline Plate length & 270 & $\mathrm{~mm}$ \\
\hline$m_{0}$ & 42 & $\mathrm{~g}$ \\
\hline$p$ & $3.27 \cdot 10^{4}$ & $\mathrm{~N} / \mathrm{mm}$ \\
\hline$b_{0}$ & 21 & $\mathrm{~mm}$ \\
\hline
\end{tabular}




\section{Conclusions}

Although the measurements were carried out only for experimental reasons, without a properly controlled environment, it gave surprisingly good results. It is hard to derive the curvature from a low precision set of points, since it is a complex expression, containing derivatives, however the results are quite promising. On one hand, the method in its current state is not adequate for the precise measurement of the curvature, but it does not exclude the possibility of further experiments. On the other hand, it is an adequate method of measuring the bent shape of a plate.

\section{Acknowledgements}

This work has been supported by the National Research, Development and Innovation Office (NKFI) under grant No.134303.

\section{References}

[1] Vladimir K.: Curvature in Digital 2D Images. International Journal of Pattern Recognition and Artificial Intelligence, 15/7. (2001) 1183-1200. https://doi.org/10.1142/S0218001401001283

[2] Coeurjolly D., Miguet S., Tougne L.: Discrete Curvature Based on Osculating Circle Estimation. In: Visual Form 2001. $4^{\text {th }}$ International Workshop on Visual Form, IWVF4 Capri, Italy, May 28-30, 2001. Proceedings, 303-312. https://doi.org/10.1007/3-540-45129-3_27

[3] Dalle D.: Comparison of Numerical Techniques for Euclidean Curvature. Rose-Hulman Undergraduate Mathematics Journal, 7/1. Art.12.

[4] Belyaev A. G.: A Note on Invariant Three-Point Curvature Approximations, 数理解析研究所講究録, 1111. (1999) 157-164. (letöltve: 2021.02.15) https://repository.kulib.kyoto-u.ac.jp/dspace/bitstream/2433/63333/1/1111-16.pdf

[5] Máté P., Szekrényes A.: Vékonylemez hengerítési folyamatának numerikus modellezése. Müszaki Tudományos Közlemények, 13. (2020) 133-136. https://doi.org/10.33895/mtk-2020.13.24 